\title{
ANTIPARASITÁRIO E A VITAMINA A: SUA ATUAÇÃO NO COMBATE AO PARASITISMO POR ASCARIDÍASE
}

\author{
Cleonice Duarte da Silva Costa ${ }^{1}$ \\ Kely Alves de Oliveira ${ }^{2}$ \\ Leonardo Morais Dias ${ }^{3}$ \\ Patrícia Rosa dos Santos ${ }^{4}$ \\ Raquel Cristina Freitas Nascimento ${ }^{5}$
}

\begin{abstract}
RESUMO: Os parasitas de humanos o helminto Ascaris lumbricoides, geralmente conhecido como a lombriga causa um tipo de parasitose intestinal, a ascaridíase, causada em toda espécie humana e principalmente em crianças. Em alguns países é feito um planejamento no sentido de empregar programas que visam o controle desses parasitas intestinais, que tanto acometem o bem estar da população, principalmente aqueles que são de classe baixa. O objetivo principal deste relatório foi identificar como este medicamento antiparasitário, em específico, a Ivermectina age no corpo humano, como é metabolizado, para quais doenças parasitárias ou doenças em específico ela está sendo empregada e como ela afeta a população humana, considerando um melhor entendimento desse grave problema a saúde pública. Sabemos que esse fármaco é de extrema relevância, possui atividade antiviral e antiparasitário é usado no tratamento de vários tipos de infestações por vermes e parasitas, ultimamente seu consumo tem aumentado muito de forma exacerbada,
\end{abstract}

\footnotetext{
I ORCID: https://orcid.org/oooo-oooI-766I-34I8. Formada no curso técnico de magistério e contabilidade, acadêmica do curso de Farmácia da UNIP, Brasil, e-mail: cleoduartesilvacosta@gmail.com.

2 ORCID: https://orcid.org/oooo-ooo3-3872-682X. Graduada em Administração de Empresas, com especialização em Gestão de Pessoas, acadêmica do curso de Farmácia da UNIP, Brasil, e-mail: kelyddiniz@yahoo.com.br.

3 ORCID: https://orcid.org/oooo-ooo2-860o-4509. Formado no curso técnico de enfermagem pelo Senac-GO, acadêmico do curso de Farmácia da UNIP, Brasil, e-mail: leolorio2i978@hotmail.com.

4 ORCID: https://orcid.org/oooo-0oo2-5144-8302. Formada no Curso técnico de Análise Clínica, atua há mais de ro anos na área, acadêmica do curso de Farmácia da UNIP, Brasil, e-mail: paty.prdsrosao9@gmail.com.

5 ORCID: https://orcid.org/oooo-ooo2-5053-337X. Graduada em Ciências Biológicas-Modalidade Médica pelo Centro Universitário Barão de Mauá de Ribeirão Preto- SP, com especialização em Citologia Clínica pela Universidade Federal de Goiás - Faculdade de Farmácia, acadêmica do curso de Farmácia da UNIP, Brasil, e-mail: raquelcristinafreitas2or8@gmail.com.
} 
mesmo sem uma comprovação científica para tal fim, devido a atual pandemia mundial. Utilizamos para agregar a pesquisa uma molécula de Vitamina A, no sentido de fortalecer a molécula da Ivermectina, visto que, crianças acometidas por esse parasita sofre por hipovitaminose A, causando até um depauperamento físico e mental. Sabendo que para um medicamento se tornar recomendado, confiável e legítimo, precisa passar pelo método analítico de validação. Logo, a validação é de extrema relevância para assegurar a confiabilidade dos resultados encontrados.

Palavras-chave: Ascaridíase. Antiparasitário. Ivermectina. Vitamina A.

ABSTRACT: The parasites of humans, the helminth Ascaris lumbricoides, generally known as the roundworm, cause a type of intestinal parasitosis, ascariasis, caused in all human species and mainly in children. In some countries, a plan is made to employ programs aimed at controlling these intestinal parasites, which affect the well-being of the population so much, especially those who are from the lower class. The main objective of this report was to identify how this antiparasitic drug, in particular, Ivermectin acts on the human body, how it is metabolized, for which specific parasitic diseases or diseases it is being used for and how it affects the human population, considering a better understanding public health from this serious problem. We know that this drug is extremely relevant, has antiviral and antiparasitic activity and is used in the treatment of various types of infestations by worms and parasites, lately its consumption has increased dramatically, even without scientific proof for this purpose, due to the current world pandemic. We used to aggregate the research a molecule of Vitamin A, in order to strengthen the molecule of Ivermectin, since, children affected by this parasite suffer from hypovitaminosis A, causing even a physical and mental depletion. Knowing that for a drug to become recommended, reliable and legitimate, it needs to go through the analytical method of validation. Therefore, validation is extremely important to ensure the reliability of the results found.

Keywords: Ascariasis. Antiparasitic. Ivermectin. Vitamin A.

\section{INTRODUÇÃO}

Determinadas parasitoses estão entre as doenças mais existentes no mundo, atingindo milhões de pessoas. A prevenção depende de diversos fatores como: medidas ecológicas, sanitárias, isto é, também como o emprego de fármacos antiparasitário eficazes e seguros (Fraya, 1997).

Dentre as espécies de helmintos mais frequentes está o Ascaris lumbricoides, conhecido como lombriga, muito comum na espécie humana, especialmente em crianças em idade escolar. (REY, 2002; CIMERMAN, 2005) 
A ascaridíase é uma infecção pelo nematódeo Ascaris lumbricoides, cilíndrico, possui cerca de 20 a $40 \mathrm{~cm}$ quando adulto e a fêmea é maior e mais forte que o macho. É encontrado em vários países, contudo alguns fatores tipo condições ambientais, climáticas e também o nível socioeconômico, favorecem o surgimento. Esse parasita acomete cerca de um quarto da população mundial e é a helmintíase mais comumente. Sendo mais comum na América Latina, África e Ásia, especialmente em regiões mais povoadas e com condições de saneamento básico insatisfatória. O que contribuem para a proliferação são o costume de usar fezes como adubação, defecar no chão e não higienizar as mãos antes de levá-la a boca.

Percebe-se que mesmo através de projetos e campanhas realizadas, sabe-se que os níveis de parasitismo permanecem altos, sobretudo em crianças menores de 12 anos. Outro atenuante da disseminação desse parasita é a resistência dos seus ovos, que se sustentam mesmo em condições ambientais adversas, como congelação e ressecamento.

O período de incubação entre a ingestão do ovo e a chegada do parasita adulto ao lúmen intestinal dura cerca de dois meses. Durante esse período as larvas passam por vários órgãos, como fígado e pulmões. Em casos esporádicos, podem provocar irritação pulmonar com hemorragias e hemoptise (tosse com sangue). Juntamente com outros sintomas nessa etapa como tosse, falta de ar e febre baixa.

Assim que o parasita chega ao intestino, ele se alimenta do bolo alimentar. Alguns sintomas incluem como: náusea, vômito, diarreia, dor abdominal, perda de apetite, febre, ruído ao respirar.

O tratamento para ascaridíase é feita por um antiparasitário como a ivermectina, azólicos, mebendazol ou o albendazol em dose única, deve ser repetido após algumas semanas para matar larvas que possam estar migrando.

\section{I.I Ivermectina}

$\mathrm{Na}$ década de 7o, o pesquisador Satoshi Omura, realizou análises com bactérias presentes no solo e, por meio da criação de culturas de bactérias do gênero Streptomyces, 
uma classe isolada de produtos de fermentação de um actinomiceto, conseguiu identificar que várias dessas culturas produziam substâncias tóxicas para outros organismos.

A partir da pesquisa de Omura, o pesquisador William Campbell começou a avaliar a eficácia dessas culturas. Uma das espécies descobertas, a S. avermitilis, produz a avermectina que combate, de forma eficiente, diversos parasitas de animais. Em seguida, o composto foi quimicamente modificado para a criação da Ivermectina, sendo um derivado semi-sintético, que é uma substância mais eficiente no tratamento e com amplo espectro de ação.

A droga, descoberta na década de 70 se mostrou ser um excelente antiparasitário. Por sua vez, na década de 80 deram início os primeiros testes clínicos em humanos usando Ivermectina para tratar a oncocercose. O fármaco mostrou-se seguro e eficaz.

Em 1987 a Ivermectina foi doada pela fabricante Merck er CO. Inc. (sob a marca "Mectizan") e em 1988 a distribuição livre da droga terapêutica foi agrupada nas atividades do Programa de Controle da Oncocercose.

Em 1991, nas Américas foi criado um Programa para Eliminação da Oncocercose (OEPA). A doença que no Brasil se restringe as áreas indígenas habitadas por povo yanomamis, abrange as fronteiras amazônicas do Brasil e Venezuela (Ministério da Saúde, 199I).

Em 1994 e 1995 o Programa das Nações Unidas para o Desenvolvimento (PNUD), o Banco Mundial, a Organização Mundial de Saúde (OMS), o Programa Especial para Pesquisa e Treinamento em Doenças Tropicais (TDR) e o Programa Africano de Controle da Oncocercose (APOC), começaram a desenvolver um mecanismo inovador de Tratamento com Ivermectina, a estratégia baseava-se na participação da comunidade, foi lançado com o intuito de controlar a doença em 19 países onde a doença era endêmica.

Com o objetivo de entregar a Ivermectina para mais ou menos de 90 milhões de pessoas, uma vez por ano, protegendo 120 milhões de pessoas em risco de 
contaminação e prevenção de mais de 40 mil casos de cegueira por ano. Estima-se que cerca de 40 milhões de pessoas tenham sido protegidas, 600 mil casos de cegueira tenham sido impedidos, 18 milhões de crianças nascido livres da ameaça da doença e 25 milhões de hectares de terra tornados seguros para a produção agrícola.

Embora em 2015 os pesquisadores foram presenteados com o prêmio Nobel, seu descobrimento é antigo e, já auxiliou milhões de pessoas, especialmente nas Américas e na África, onde a situação era mais grave.

Sob condições ambientes, a Ivermectina apresenta-se como um pó branco e cristalino e, livremente solúvel em diclorometano e solúvel em etanol, podemos citar sua baixíssima solubilidade em água. Dentre as características físico-químicas de relevância para a molécula, temperatura de fusão entre $155^{\circ} \mathrm{C}$ e $157^{\circ} \mathrm{C}$, coeficiente de partição octanol-água $(\log \mathrm{P})$ de 5,83 e (pKa) de 12,47 e comportamento de ácido fraco.

Em relação a sua farmacocinética, é bem absorvida no trato gastrointestinal, até que chega ao seu alvo, efetua sua ação e se transforma em um produto a ser eliminado, é metabolizado pelo fígado, CYP45o, possui meia-vida biológica de 18 horas, sua ligação plasmática é de 93\%, sua via de administração é oral.

Ao finalizar sua tarefa, ela é excretada pela via fecal e $<1 \%$ urina renal, em um período estimado de 12 dias. Quanto à farmacodinâmica, seu mecanismo de ação, age contra os parasitas e vermes por meio da paralisação tônica, atua imobilizando suas musculaturas. Isso faz que eles morram e sejam eliminados do organismo.

Por sua vez a Ivermectina é sintetizada a partir da avermectina e é constituída por uma mistura de homólogos que não deve conter menos que 80\% de seu homólogo Bia (5-O-dimetil-22,23-di-hidroavermectina $A_{1 a}$ ), que tem fórmula molecular $\mathrm{C}_{48} \mathrm{H}_{74} \mathrm{O}_{14}$ e massa molecular de $875 . \mathrm{I} \mathrm{g} / \mathrm{mol}$ e não mais de $20 \%$ de seu homólogo $\mathrm{BIb}$ (5-O-dimetil-25-(I-metilpropil) -22,23-di-hidro-25-(I-metil-etil) avermectina $A_{1 a}$ ), de fórmula molecular $\mathrm{C}_{47} \mathrm{H}_{72} \mathrm{O}_{14}$ e massa molecular $861.07 \mathrm{~g} / \mathrm{mol}$.

Sua composição é feita por (butil-hidroxianisol, ácido cítrico, celulose microcristalina, lactose, laurilsulfato de sódio, dióxido de silício e estearato de magnésio). 
Figura I- Estrutura Química da Ivermectina

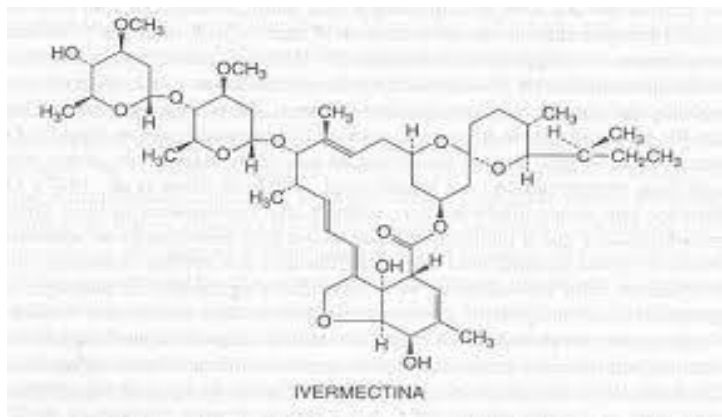

Fonte: Google

\subsection{Algumas das doenças onde a Ivermectina tem a mesma finalidade}

A Ivermectina é um fármaco do grupo dos antiparasitários com ação em vários tipos de infestações por vermes, parasitas, ácaros e os nematódeos.

Dada a ampla utilização desse fármaco por mais de 40 anos, ele é considerado muito seguro. Contudo, é relevante que você o utilize de forma racional, isto é, de acordo, com a forma adequada de doses e por tempo determinado.

A Ivermectina pode ser empregada nas seguintes infestações:

a) Estrongiloidíase intestinal: causada por Strongyloides stercolaris, manifestações incluem exantema e sintomas pulmonares (incluindo tosse e sibilos), eosinofilia e dor abdominal com diarreia;

b) Oncocercose: cuja origem é o parasita Onchocerca volvulus, a doença é transmitida por frequentes picadas de mosquitos pretos infectados. É mais comum em pessoas que vivem em vilarejos africanos remotos. Em regra, os sintomas não aparecem por até um ou dois anos após a infecção. Nódulos na pele e coceira podem se desenvolver. Infecções oculares podem levar a mudanças de visão e cegueira;

c) Elefantíase (filariose): cuja origem é o Wurchereria brancrofti, é uma doença parasitária, que consegue atingir os vasos linfáticos e promove uma reação inflamatória, causando uma obstrução do fluxo de linfa e levando ao acúmulo de líquido e inchaço em alguns órgãos, como braço, testículo e etc; 
d) Lombriga (ascaridíase): causada pelo Ascaris lumbricóides, é uma doença parasitária intestinal causada por um nematoide, que acomete a população, especialmente aqueles de baixa renda e que vivem em locais com saneamento básico precário;

e) Sarna (escabiose): causada por um ácaro chamado Sarcoptes scabiei, também conhecida como sarna humana, é uma doença de pele que é facilmente transmitido de pessoa para pessoa, através do contato físico, e raramente por roupas ou outros objetos compartilhados, e que leva ao aparecimento de bolhas e placas vermelhas na pele que coçam muito, sobretudo à noite;

f) Piolhos (Pediculose): causada por um parasita Pediculus humanus, os piolhos são insetos pequenos, sem asas, que se alimentam de sangue. A transmissão ocorre pelo contato direto ou pelo uso de bonés, chapéus, escovas de cabelo, pentes ou roupas de pessoas contaminadas.

1.3 Medicamentos existentes no mercado para tratar essa finalidade e suas moléculas

Fazendo parte da lista de medicamentos essenciais da Organização Mundial de Saúde revelou em 2017 uma lista com os medicamentos mais seguros e eficazes fundamentais num sistema de saúde, se administrado de acordo com as doses autorizadas:

$>\quad$ Ivermectina: Ivermec, Iverneo, Leverctin, Revectina, Plurimec e Vermectil;

> Albendazol: Zentel, Zolben, Parasin, Albel, Mebenix e Albentel;

> Tiabendazol: Thiaben, Tiadol, Micosbel,Tiaplex,Dazotil e Foldan;

> Cambendazol: Cambem e Exelmin;

$>\quad$ Nitazoxanida: Annita;

> Mebendazol: Pantelmin, Licor de Cacau Xavier, Necamin e Multielmin;

$>\quad$ Levamisol: Ascaridil;

> Dietilcarbamazina: Hetrazan, Banocide, Notézine, Caricide, Carbilazine; 
Oxamniquina: Mansil;

$>\quad$ Niclosamida: Atenase;

> Metronidazol: Flagyl, Helmizol, Neo Metrodazol, Terconazol, Rozex;

> Pamoato de Pirantel: Combantrin e Ascarical;

$>\quad$ Praziquantel: Cisticid e Cestox.

Figura 8- Molécula do Levamisol




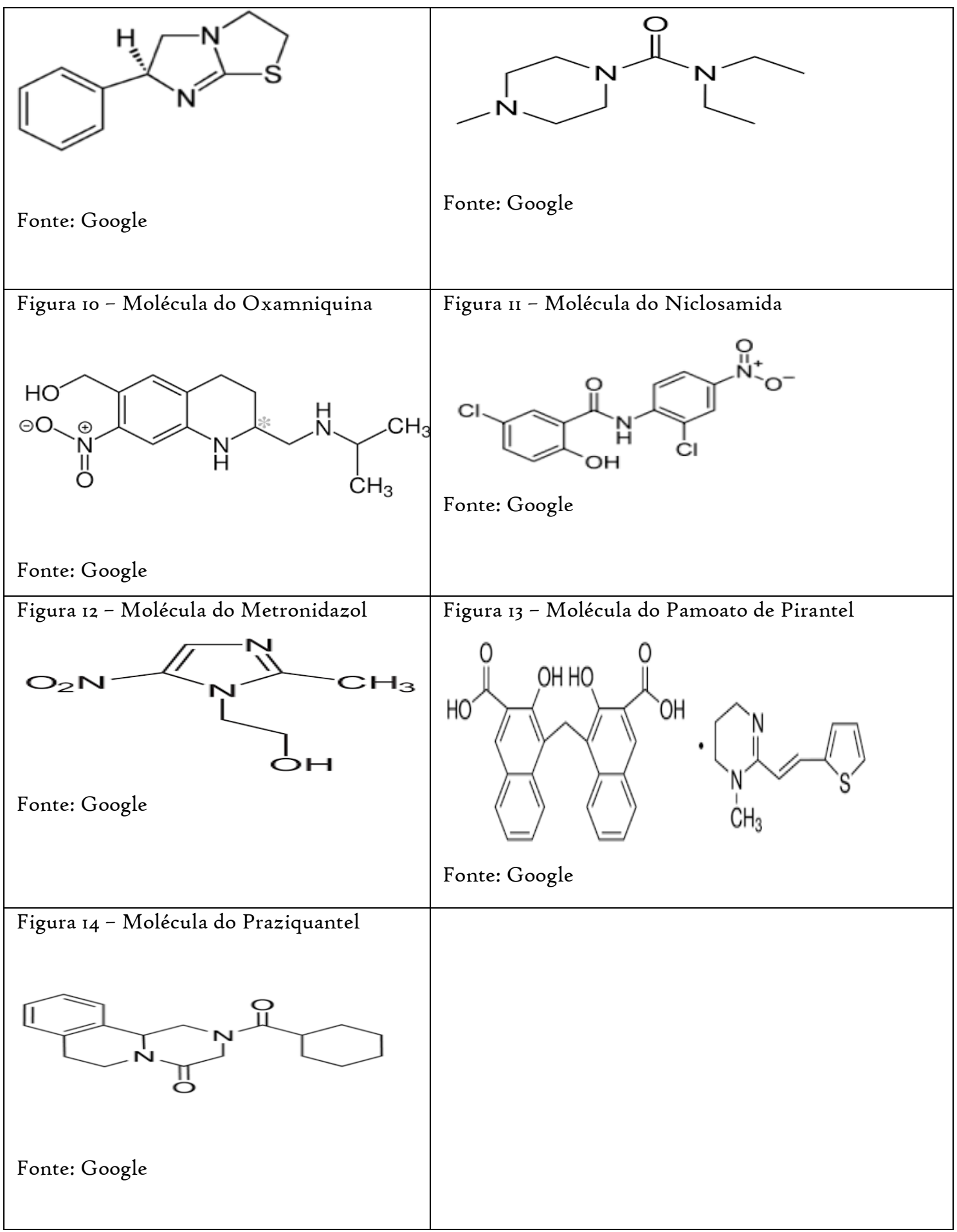

1.4 Limitações dos antiparasitários disponíveis, seus efeitos indesejáveis e preços 
Alguns antiparasitários desapareceram das farmácias, inclusive a Ivermectina, com isso dificultou a vida de muitos pacientes que precisam fazer uso deste medicamento na prevenção ou tratamento de doenças, como por exemplo, a escabiose, (sarna humana), que é causada por ácaro que resulta em lesões com coceiras intensas, se não tratada pode ser transmitido pelo contato íntimo ou, secundariamente de materiais como lençol, estofados e etc. Devido ao momento atual pandêmico em que a sociedade se encontra, houve uma procura desenfreada deste medicamento e outros que possuem a mesma finalidade.

Vale ressaltar que a Ivermectina é o tratamento mais eficiente para a escabiose e que a falta dele exige que pacientes tenham que mandar manipular uma loção, algo demorado e que nem sempre é fácil de encontrar.

Os antiparasitários apresentam como efeitos indesejáveis: náuseas, vômitos, diarreia, dores abdominais, reações alérgicas (broncoespasmo e urticária), tonturas, confusão mental, descoordenação motora, angioedema e síndrome de Stevens-Johnson (descritos raramente). Está contra indicado no período de gravidez e aleitamento, em casos de disfunção hepática, epilepsia, doença neurológica e doença renal grave.

Não são medicamentos de alto custo, mas devido a pandemia houve um grande aumento nos preços, por ser os mais procurados no momento.

\subsection{Posologia da Ivermectina e possíveis reações adversas}

Embora esses medicamentos sejam bastante seguros, é importante que eles sejam utilizados somente sob orientação médica. Além disso, somente o médico poderá identificar que tipo de parasita se busca eliminar, qual seria a dose ideal e, sobretudo, se haverá, ou não, necessidade de repetição do tratamento ou de uma terapia que combine outros medicamentos. Em regra, o remédio começa a fazer efeito a partir de 4 horas de seu uso.

A posologia recomendada para o tratamento de parasitoses costuma ser em doses únicas oral, por quilo e peso corpóreo, geralmente não são necessárias doses 
secundárias. Contudo, deve-se verificar com o médico se necessário repetir a dose de acordo com exames laboratoriais.

Em infestações externas, pode ser administrada aplicada na pele. $O$ contacto com os olhos deve ser evitado. Em doses mais altas ou em tratamento por período longo, há risco de danos renais e hepáticos, hepatite medicamentosa, coma, convulsão e até morte.

Os efeitos secundários mais observados são olhos vermelhos, pele seca e sensação de queimadura. Não é claro se a sua administração é segura durante a gravidez, embora seja provavelmente aceitável o seu uso durante a amamentação. Bem tolerada por adultos e idosos, só pode ser usada por crianças acima dos 5 anos e $15 \mathrm{~kg}$.

É contraindicada para pessoas que sejam alérgicas, com meningite ou algumas enfermidades do SNC.

Algumas possíveis reações adversas podem aparecer como: diarreia, náusea, dor abdominal, falta de apetite, constipação, vômito, tontura, sonolência, vertigem, tremor, febre, coceira, lesões de pele, urticária.

Outras manifestações podem ser observadas nos casos de oncocercose, mas a doença é mais comum em países africanos.

\section{HIPÓTESE}

O medicamento experimental proposto tende a ser mais eficaz, do que atualmente se encontra-se no mercado, visto que, propõe a realizar a adição de duas moléculas como antiparasitário e vitamina $\mathrm{A}$, necessárias para o tratamento da Ascaridíase.

\section{OBJETIVO}

Encontrar um novo fármaco antiparasitário que seja associada com nutrientes necessários, devido a deficiência das infecções parasitárias por Ascaris lumbricoides 
sobre o sistema imunológico, com vistas a uma melhor abrangência desse grave problema de saúde pública.

\section{JUSTIFICATIVA}

A proposta do tema surgiu da necessidade de se discutir o parasitismo por Ascaris lumbricoides, visto que a exposição por esse parasita oferece grande risco à saúde da população.

O Ascaris lumbricoides consome grandes quantidades de proteínas, carboidratos, lipídios, vitamina $\mathrm{A}$ e $\mathrm{C}$, levando principalmente as crianças à subnutrição e ao depauperamento físico e mental.

Por sua vez, a falta de algumas vitaminas traz problemas graves a saúde, é conhecida por avitaminose ou hipovitaminose.

Percebemos que as crianças menores de 12 anos, entre os grupos de baixo socioeconômico que se alimentam muito mal e vivem em condições sanitárias pouco satisfatórias que são as mais afetadas e são as mais propensas a sofrer este contágio, por isso, a alimentação adequada e as condições de saneamento básico, com certeza, se tornariam imprescindível nesse processo.

Mas como nem sempre isso é possível, a lavagem das mãos com sabão também protege contra a infecção. Em áreas onde mais de $20 \%$ da população está infectada, recomenda-se o tratamento de todos em intervalos periódicos, devido as possíveis reinfecções. Como não existe vacina, a OMS, Organização Mundial de Saúde, recomenda o uso das medicações como Ivermectina, nitazoxanida, albendazol, mebendazol, levamisol ou pamoato de pirantel, entre outros agentes.

Entretanto, a necessidade de um fármaco no sentido de agregar um antiparasitário a uma molécula de Vitamina A, tornaria a criança mais forte e resistente a esses parasitas. Com isso facilitaria a administração, visto que, a criança iria estar tomando um medicamento que estará compondo duas moléculas.

Por sua vez, a vitamina A ou Retinol é um micronutriente pertencente ao grupo das vitaminas lipossolúveis, podendo ser encontrada no tecido animal sob a forma de 
retinóides ou como pró-vitamina em tecidos vegetais, sob a forma de carotenoide. No organismo humano a vitamina A pode ser encontrada de três formas, o retinol, retinal e o ácido retinóico, sendo o último o principal e mais abundante metabólito intracelular em mamíferos.

São moléculas fundamentais para nossa saúde e encontradas em nossos alimentos. Apesar de serem essenciais, as vitaminas não precisam ser ingeridas em grande quantidade, porém são essenciais para garantir o funcionamento adequado do nosso organismo. Atuam, como catalizadores de reação dentro dele. São importantes na transformação de energia, antioxidantes e essenciais para o funcionamento dos vários sistemas do corpo, inclusive o nosso sistema imunológico.

\section{MATERIAL E MÉTODOS}

\section{I Rota sintética da molécula de Vitamina A}

A síntese de um fármaco é de extrema relevância para a química orgânica, uma vez que admite a construção de moléculas em seus níveis de complexidade. Esse desenvolvimento apresenta características particulares, pois além de justificar uma sequência de etapas sintéticas, dispensando atenção ao grau de pureza e a escala de reação, visando obter rendimentos satisfatórios. A vitamina A apresenta algumas propriedades como: fórmula molecular $\mathrm{C}_{20} \mathrm{H}_{30} \mathrm{O}$, massa molar $286.456 \mathrm{~g} / \mathrm{mol}$, ponto de fusão $6 \mathrm{I}-63^{\circ} \mathrm{C}$, ponto de ebulição $120-125{ }^{\circ} \mathrm{C}$, praticamente insolúvel em água, apresenta uma solubilidade solúvel em solventes orgânicos apolares, possui alguns compostos relacionados como: o ácido retinóico, o alfacaroteno e o betacaroteno.

A síntese da Vitamina A se dá por meio da ação da enzima betacaroteno-15,15dioxigenase, que em mamíferos possui uma atividade máxima na mucosa intestinal. Por ser uma enzima exclusiva de animais, o retinol não está presente em tecidos vegetais, sendo assim, é falsa a afirmação de que os vegetais são fontes de Vitamina A. Logo, as frutas são fontes de pró-vitamina A, que são os carotenoides. 
A formação do retinol pela ação da enzima sobre os carotenoies na parede do intestino é absorvido e se soma com o retinol pré-formado consumido por meio de alimentos de origem animal, principalmente carnes, leite e derivados e ovos.

Devido à presença de dois anéis beta-ionona na molécula de beta-caroteno, este pode ser $100 \%$ transformado em retinol, isto é, Img de beta-caroteno produz Img de Retinol. Contudo, nem todo o carotenoide ingerido sofre transformação em retinol em nosso organismo, para que essa conversão acontece e se torne eficiente, depende de vários fatores, por exemplo, alimentação adequada, o estado nutricional do indivíduo, e mesmo assim, é sabido que a conversão depende de cada organismo.

Para que aconteça a biodisponibilidade dos carotenoides no organismo para a conversão na vitamina $\mathrm{A}$, são distribuídos na fase lipídica no período da alimentação que, sob a ação de enzimas e ácidos digestivos, sofre a conversão em micelas. $\mathrm{Na}$ fase aquosa as micelas são distribuídas na forma de uma suspensão estável. Conduzidas à mucosa intestinal, uma fração dos carotenoides sofre a ação da enzima que é responsável para converter em retinol, sendo absorvido e conduzido até o fígado onde é armazenado. Parte dos carotenoides sofre absorção sem modificações e circulam pelo organismo através do plasma.

Seu transporte se dá por meio da absorção das vitaminas quando existe lipídeos (gordura), bile e suco pancreático. Após a absorção no intestino, estas são armazenadas nos tecidos. Sendo obtida por meio de alimentos de origem animal, a vitamina A é consumida sob a forma de palmitato de retinila. Com o intuito de ser absorvido no intestino delgado estes ésteres sofrem uma hidrólise catalisada pela enzima retinil éster hidrolase, localizada na superfície externa da membrana celular da mucosa intestinal. Por conseguinte, o retinol produzido é absorvido através de células da mucosa por processo ativo, para o interior da célula, onde volta a combinar-se com um ácido graxo, geralmente o palmitato ou o estearato.

O éster de retinil é transportado para os quilomícrons através do sistema linfático e da corrente sanguínea até o fígado, onde é armazenado. As reservas de vitamina A no fígado são hidrolisadas pelas enzimas em retinol livre que é 
transportado por um complexo protéico ligante de retinol (Retinol Binding Proteins $\mathrm{RBP}$ ) aos tecidos do organismo onde existirem necessidades metabólicas. A vitamina A na forma de retinol é tóxica ao organismo humano, por este motivo sempre que é transportada aos tecidos deve estar ligada a RBP e quando no meio intracelular encontra-se ligada a proteínas citoplasmáticas ligadoras de retinol.

A toxicidade da vitamina A pode ser causada pela ingestão de doses superioras. A toxicidade aguda causa exantema, dor abdominal, aumento da pressão intracraniana e vômito. A toxicidade crônica provoca exantema, aumento da pressão intracraniana, cabelo esparso e grosseiro, pele seca e áspera e artralgia; o risco de fraturas é maior, especialmente em idosos. Na maioria dos casos o diagnóstico é baseado em achados clínicos.

Figura 15 - Molécula da Vitamina A

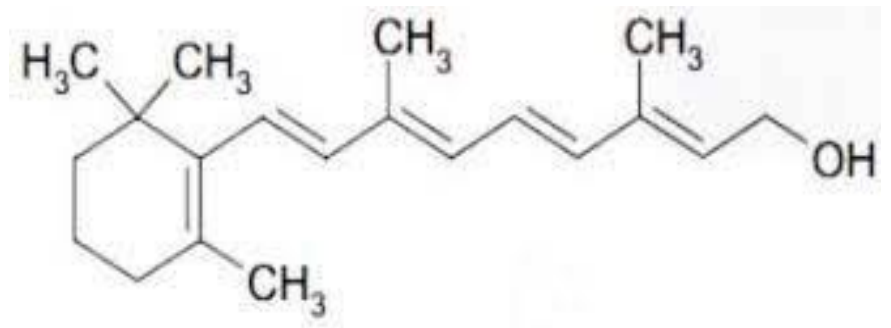

\section{VITAMINA A (RETINOL)}

Fonte: Google

\subsection{Metodologia da incorporação do princípio ativo em uma nova forma farmacêutica}

A formulação de medicamentos inclui estudos de pré-formulação para ter conhecimento de características físicas, químicas e biológicas de todas as substâncias ativas e dos adjuvantes a serem utilizados na fabricação do medicamento. Deste modo, o desenvolvimento de novos medicamentos abrange a elaboração de novas formas farmacêuticas (ALLEN, POPOVICH, ANSEL, 2013). 
De acordo com a RDC 3 I de 20Io, formas farmacêuticas são o estado final de apresentação dos fármacos após uma ou mais operações feitas com ou sem a adição de quantidade suficiente de excipientes adequados, a fim de facilitar a sua utilização e obter o efeito terapêutico desejado, com características apropriadas para uma determinada via de administração (BRASIL, 2010).

Uma grande vantagem na utilização de formas farmacêuticas sólidas em relação a líquidas, por exemplo, é a administração de doses corretas, pois não há a necessidade do uso de dispositivos de medidas. Além disso, é possível trabalhar o tamanho, a forma do medicamento e estabelecer como será realizada a liberação do princípio ativo (ALLEN, POPOVICH, ANSEL, 2013).

Desde a década de 8o, os comprimidos, são os mais produzidos e consumidos, podendo ser classificados quanto a liberação do fármaco em imediata ou controlada. Algumas vantagens existem em relação a essas formas sólidas, como exemplo, a diversidade no tamanho, forma e peso, o que facilita a ingestão e otimizar a farmacoterapia, apresentando melhor controle microbiológico e de estabilidade, e são produzidos, embalados e transportados de forma mais econômica (PASCHOAL, 2008).

Adotamos a administração por via oral por meio de comprimidos, visto que, é a mais empregada na administração de medicamentos. Normalmente o fármaco permanece no estômago em média por 2 a 4 horas (tempo de esvaziamento gástrico), logo, no intestino delgado permanece durante 4 a io horas.

Para essa finalidade a técnica que utilizamos foi a associação molecular, por meio da adição molecular, onde utilizamos a molécula da Ivermectina com a molécula da Vitamina A. Assim, obtemos fármacos que apresentem propriedades mais desejáveis da atividade terapêutica devido as seguintes ações: especificidade, facilidade de administração, estabilidade, aumento da biodisponibilidade, diminuição da toxicidade, prolongamento da ação e aumento da seletividade, mediante a escolha de transportador adequado, geralmente de caráter lipofílico. 


\subsubsection{Associação Molecular - Adição Molecular}

Figura i6 - Molécula da Ivermectina
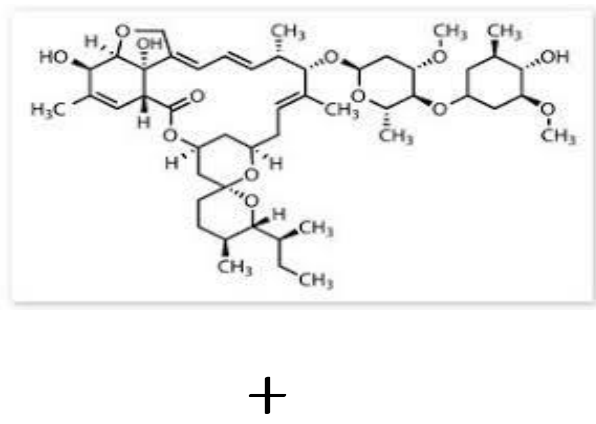

Figura 17 - Molécula da Vitamina A<smiles>CC1=C(/C=C/C(C)=C/C=C/C(C)=C/CO)C(C)(C)CCC1</smiles>

Fonte: Google

\subsection{Metodologia de Controle de Qualidade Qualitativo}

O controle de qualidade de medicamentos visa por meio das Boas Práticas de Fabricação, no que se refere à amostragem, especificações, ensaios, métodos de organização, documentação e procedimentos de liberação que garantem que os ensaios sejam indispensáveis, e que os medicamentos não sejam liberados e autorizados para a venda e uso até que tenham garantia da sua eficácia e confiabilidade (PARISOTTO et al., 2005).

Por sua vez, o controle de qualidade de medicamentos tem provocado cada vez mais apreensões, induzindo assim a várias pesquisas e análises, tornando indispensável o desenvolvimento de métodos analíticos satisfatórios e eficazes garantindo a qualidade de medicamentos (PARISOTTO et al., 2005).

O método analítico precisa passar pela validação para se tornarem confiáveis e legítimos (BRASIL, 2003). Sendo assim, a validação é de extrema relevância no que 
tange a processos de análises de medicamentos, isto é, assegura a confiabilidade dos resultados encontrados (PASCHOAL, 2008).

Algumas características de controle de qualidade qualitativa são: específico, confiável, baixo custo, fácil execução, teste de solubilidade e ensaios de pureza.

Por sua vez as técnicas cromatográficas, principalmente a Cromatografia Líquida de Alta Eficiência, são as técnicas mais utilizadas no controle de qualidade pelas indústrias farmacêuticas.

\subsection{Resultados}

Diante da magnitude e da gravidade, onde percebemos que a hipovitaminose $\mathrm{A}$ se trata de um problema de saúde pública e as parasitoses são as doenças mais perniciosas do mundo, segundo a Organização Mundial de Saúde, principalmente em países que estão em desenvolvimento e subdesenvolvimento. Nesse caso, a vantagem de obter uma associação molecular por meio da adição das moléculas de um antiparasitário com a molécula da Vitamina $\mathrm{A}$, visto que, as crianças são as que se encontram mais suscetível a infecção, estar tomando apenas um comprimido dentro da formulação ou uma dose, onde seu organismo terá uma aceitação maior. Para uma abordagem terapêutica consumirá o nível seguro de ingestão de vitamina A recomendado pela OMS, associada a mesma recomendação de um antiparasitário, e estaria ao mesmo tempo, controlando as duas doenças que mais acometem as crianças principalmente aquelas de baixa renda.

\subsection{DISCUSSÃO}

O estudo da correlação entre as parasitoses e a deficiência da vitamina $A$, assume uma grande relevância principalmente nas grandes áreas endêmicas de parasitoses que se concentram em regiões tropicais, acometendo $30 \%$ da população mundial e é frequentemente associada a outras parasitoses por outros helmintos e protozoários. onde, ao mesmo tempo, é alta o índice de desnutrição. O tratamento se torna ineficaz contra as parasitoses nas populações que se encontram desnutridas, e 
onde não possuem melhoria nas condições humanas. Dados epidemiológicos da ascaridíase e da hipovitaminose no Brasil são de difícil conhecimento, pois não há a obrigatoriedade de notificações pelo Ministério da Saúde.

É fato que, o planejamento de novos medicamentos requer, juntamente com os estudos de pré-formulação contribuir para sanar a necessidade de um público que possa se beneficiar com a inovação, como as crianças, por exemplo, os quais requerem uma maior adequação quanto à dosagem e o modo de administração (GIL, 2006).

\section{CONCLUSÃO}

Conclui-se que a Ascaris lumbricoides é a espécie mais dominante dos parasitas intestinais e o grupo mais acometido por esse helminto é o das crianças, principalmente aquelas de baixa renda. A infestação desse parasita é marcada por locais onde o saneamento básico está em condições precárias. Devem-se melhorar e estimular os hábitos de higiene das crianças, visto que, a transmissão da ascaridíase acontece na ingestão de água ou alimentos que estejam contaminados.

A ascaridíase em populações onde a ingestão de vitamina $\mathrm{A}$ e seus precursores é deficiente contribui de forma importante para a deficiência clínica de vitamina A. Por isso, se torna indispensável e de extrema relevância o conhecimento e a conscientização da população em se tratando de um problema de saúde pública, a hipovitaminose $\mathrm{A}$ e as parasitoses, que são as doenças mais nocivas do mundo, segundo a Organização Mundial de Saúde.

Programas de educação, prevenção e tratamento das infecções parasitárias, a fim de diminuir seus elevados índices, principalmente na população de crianças. $O$ objetivo é propor medidas de manejo que sejam viáveis na sua aplicação e sustentáveis em logo prazo.

Por sua vez, a Ivermectina se trata de um antiparasitário recomendado e eficaz devido ao seu grande espectro de ação.

Logo, a junção da Ivermectina com a molécula proposta a Vitamina $\mathrm{A}$, tende a ser vantajosa no sentido de tratar a ascaridíase e a hipovitaminose A, que por sua vez, 
irá atuar contra os parasitas e na manutenção e no funcionamento adequado do sistema imunológico, mantendo saudáveis as mucosas e também atuando como barreiras de proteção contra infecções.

\section{REFERÊNCIA BIBLIOGRÁFICA}

ALLEN JR., L.V.; POPOVICH, N. G.; ANSEL, H. C. Formas Farmacêuticas e Sistemas de Liberação de Fármacos; tradução Elenara lemos-Senna et al. - 8a․ Edição - Porto Alegre; Artmed, 2007.

ANDRADE, E. C. et al. Parasitoses Intestinais: Uma revisão sobre seus aspectos sociais, epidemiológicos, clínicos e terapêuticos. Revista APS, Juiz de Fora, Minas Gerais, 2010.

BRASIL. Ministério da Saúde. Agência Nacional de Vigilância Sanitária. Consulta Pública - CP n ${ }^{\circ}$ ir6, de 23 de dezembro de 2010.

BRASIL. Guia de Vigilância Epidemiológica e Eliminação da Filariose Linfática, Brasília, DF, 2009.

BRITSH, PHARMACOPÉIA. London, Her Majesty's Stationary, i98o.

CARVALHO, O. S. et al. Prevalência de helmintos intestinais em três mesorregiões do Estado de Minas Gerais. Revista da Sociedade Brasileira de Medicina Tropical, 2002.

CIMERMAN, B.; CIMERMAN, S. Parasitologia humana e seus fundamentos gerais. 2. ed. São Paulo: Atheneu, 2005 .

COUTO, A. G.; GONZÁleZ, G.O.; PETROVICK, P. R. Granulação. Caderno de Farmácia, 2000.

FREHSE, Fraya. Entre largo e praça, matriz e catedral: a Sé dos cartões postais paulistanos. Cadernos de Campo, São Paulo, 1997.

GIL, E.S.; ORLANDO, R.M. Controle Físico-Químico de Qualidade de Medicamentos. Ia. Edição. Manole, Barueri, 2006.

NEVES, D. P. Parasitologia Humana. II. ed. São Paulo: Atheneu, 2005.

PARISOTTO, G. et al. Analise exploratória aplicada no estudo de medicamentos contendo piroxicam. Revista Brasileira de Ciências Farmacêuticas, São Paulo, 2005. 
PASCHOAL, J. A. R.; RATH, S.; SILVA, F. P. DA; REYES, F. G. R. Validação de métodos cromatográficos para a determinação de resíduos de medicamentos veterinários em alimentos. Revista Química Nova, Campinas, 2008.

REY, L. Bases da Parasitologia Médica. 2. ed. Rio de Janeiro: Guanabara Koogan, 2002. 